\title{
The effects of HIF-1a overexpression on renal injury, immune disorders and mitochondrial apoptotic pathways in renal ischemia/reperfusion rats
}

\author{
Xiaoli Li ${ }^{1}$, Wenhui Chen ${ }^{1}$, Jinfang Feng ${ }^{1}$, Bo Zhao ${ }^{2}$ \\ ${ }^{1}$ Department of Nephrology, ${ }^{2}$ Department of Geriatrics, Xiangyang No. 1 People's Hospital, Hubei University of Medicine, Xiangyang, China \\ Contributions: (I) Conception and design: X Li, B Zhao; (II) Administrative support: B Zhao; (III) Provision of study materials or patients: X Li; (IV) \\ Collection and assembly of data: W Chen, J Feng; (V) Data analysis and interpretation: W Chen, J Feng; (VI) Manuscript writing: All authors; (VII) \\ Final approval of manuscript: All authors. \\ Correspondence to: Zhao Bo. Department of Geriatrics, Xiangyang No. 1 people’s Hospital, Hubei University of Medicine, Xiangyang 441000, China. \\ Email: 5210904@qq.com.
}

Background: Renal ischemia/reperfusion (RI/R) injury are a common pathogenesis of acute kidney injury, which may cause renal parenchyma damage clinically. Hypoxia-inducible factor- $1 \alpha(\mathrm{HIF}-1 \alpha)$ has protective effects on cells in regulating the metabolism, angiogenesis, erythropoiesis, and anti-apoptosis of RI/R injury. However, the specific mechanisms for HIF-1 $\alpha$ on RI/R injury are still unclear. This study aims to investigate the effects of HIF-1 $\alpha$ overexpression on renal function injury, immune disorder, and mitochondrial apoptosis in RI/R rats.

Methods: The rat model of RI/R injury was set up. The lentivirus (LV) vector of HIF-1 $\alpha$ overexpression was constructed, and then the $\mathrm{LV}$ was transfected to the model rats. The rats were randomly divided into four groups: the control group, RI/R group, RI/R + LV group, and RI/R + LV-HIF-1 $\alpha$ group for later experiments. The mRNA levels of HIF-1 $\alpha$ were detected by RT-PCR. Proteinuria, urea nitrogen, and serum creatinine levels were detected using the relative kit. Pathological damage was detected by HE staining. Apoptosis was detected by TUNEL staining. Levels of interleukin-6 (IL-6), interleukin-1 $\beta$ (IL-1 $\beta$ ), tumor necrosis factor $\alpha(\mathrm{TNF}-\alpha)$ and interleukin-10 (IL-10) were detected by ELISA. Western blotting was used to detect the protein levels of HIF-1 $\alpha$, caspase-3, caspase-9, Bax, Bcl-2, and other proteins.

Results: Compared with the control group, the mRNA and protein levels of HIF-1 $\alpha$ in the RI/R group were increased significantly $(\mathrm{P}<0.05)$. Proteinuria, urea nitrogen, serum creatinine levels were increased significantly $(\mathrm{P}<0.05)$. The levels of IL-6, IL-1 beta, TNF- $\alpha$ were increased significantly $(\mathrm{P}<0.05)$. The ratios of cleaved caspase-3/caspase-3, cleaved caspase-9/caspase- 9 , and Bax/Bcl-2 were increased significantly $(\mathrm{P}<0.05)$. There was a significant increase in apoptosis rate and renal pathological tissue damage $(\mathrm{P}<0.05)$. Compared with RI/R+LV group, the mRNA and protein levels of HIF- $1 \alpha$ in the RI/R+LV-HIF-1 $\alpha$ group were increased significantly $(\mathrm{P}<0.05)$. Proteinuria, urea nitrogen, serum creatinine levels were decreased significantly $(\mathrm{P}<0.05)$. IL-6, IL-1 beta, TNF- $\alpha$ levels were significantly decreased $(\mathrm{P}<0.05)$. IL-10 level was significantly increased $(\mathrm{P}<0.05)$. The ratios of cleaved caspase-3/caspase-3, cleaved caspase-9/caspase-9, and $\mathrm{Bax} / \mathrm{Bcl}-2$ were significantly reduced $(\mathrm{P}<0.05)$, showing that the pathological damage degree and the apoptosis rate was significantly lower.

Conclusions: HIF- $1 \alpha$ overexpression has protective effects on renal ischemia-reperfusion rats by improving pathological injury and immune function, reducing the release of inflammatory factors, and the expression of apoptotic proteins.

Keywords: Renal ischemia-reperfusion; hypoxia-inducible factor-1 $\alpha$ (HIF-1 $\alpha$ ); renal injury; mitochondrial apoptosis pathway 
Submitted Apr 14, 2020. Accepted for publication Aug 25, 2020.

doi: $10.21037 /$ tau-20-918

View this article at: http://dx.doi.org/10.21037/tau-20-918

\section{Introduction}

Renal ischemia/reperfusion (RI/R) injury are a common pathogenesis of acute kidney injury because the kidney is a highly perfused organ and is sensitive to ischemia (1). RI/R injury happens in varying degrees during the treatment of partial renal tumor resection and renal transplantation (2). During ischemia, the activity of antioxidant enzymes decreases, leading to the formation of superoxide radicals and an increase in oxidative stress during reperfusion. Superoxide radicals destroy cell components, further damaging mitochondrial function and leading to cell death. Clinically, RI/R injury may cause renal parenchyma damage because of continuous blood flow reduction and pathological process, causing acute renal failure or chronic progressive kidney injury after kidney transplantation (3). However, because of complex pathogenesis, RI/R injury is challenging to treat. Therefore, it is necessary to dig the molecular mechanisms for causing RI/R injury or preventing it.

The precise pathophysiology of RI/R is largely unclear. Multiple pathways were involved, such as generation of reactive oxygen species (ROS) and associated inducible signaling, induction of apoptosis, and persistence of hypoxia and inflammation. The most effective method to reduce $\mathrm{RI} / \mathrm{R}$ is to cool the kidney during surgery. However, there is currently no technique or drug to pretreat the kidney prior to ischemic injury. Many pharmacological reagents, such as $\mathrm{Ca}^{2+}$ channel blockers, mannitol, adenosine, and $\mathrm{N}$-acetylcysteine, have been shown to protect against RI/R in animal models, but none passed clinical trials (4).

As heterodimer, hypoxia-inducible factor- $1 \alpha$ (HIF-1 $\alpha)$ is ubiquitously expressed in mammals and plays a role in regulating cell activity, cell growth, and apoptosis to maintain normal physiological activities of cells in a hypoxic environment (5). HIF-1 $\alpha$ has protective effects on cells in regulating the metabolism, angiogenesis, erythropoiesis, and anti-apoptosis of RI/R injury (6). Studies have shown that repeated hypoxic preconditioning can ease RI/R injury via the Bcl-2 anti-apoptotic pathway activated by HIF-1 $\alpha$ (7). However, the specific mechanisms for HIF-1 $\alpha$ on RI/R injury are still unclear. This study aims to investigate the effects of HIF-1 $\alpha$ overexpression on renal dysfunction, immune disorder, and mitochondrial apoptosis pathway in rats with $\mathrm{RI} / \mathrm{R}$.

We present the following article in accordance with the ARRIVE reporting checklist (available at http://dx.doi. org/10.21037/tau-20-918).

\section{Methods}

The animal experiments in this study were approved and supervised by the Ethics Committee of the Xiangyang No. 1 People's Hospital (SYXK (Hubei) 2016-0031). Each experiment was performed in accordance with the Guide for the Care and Use of Laboratory Animals, 8th edition, published by the National Research Council (US) Committee.

\section{Experimental animals}

Forty male Sprague-Dawley (SD) rats (aged six weeks, weight $110 \pm 5 \mathrm{~g}$ ) were purchased from the Animal Experimental Research Center of Zhejiang University of Traditional Chinese Medicine, with the license number of SYXK (Zhejiang) 2018-0012. The animals were routinely housed in temperature control $\left(22 \pm 1^{\circ} \mathrm{C}\right)$ and lightcontrolled (200 lux, 12 hours light/dark cycle) animal facilities. The rats were accustomed for seven days before grouping and treatment.

\section{Reagents}

Hematoxylin-eosin stain (D006-1-1), urine protein quantitative kit (C035-2-1), creatinine determination kit (C011-2-1), urea nitrogen determination kit (C013-2-1) were purchased from Nanjing Jiancheng Bioengineering Research Institute (Nanjing, China). The TdT-mediated dUTP nick end labeling (TUNEL) apoptosis detection kit (C1091) was purchased from Beyotime Institute of Biotechnology (Jiangsu, China). Antibodies against tumor necrosis factor $\alpha(\mathrm{TNF}-\alpha)(\mathrm{ab} 6671)$, IL-6 (ab100772), interleukin-1 $\beta$ (IL-1 $\beta$ ) (ab100768), interleukin-10 (IL-10) (ab34843), Bax (ab53154), Bcl-2 (ab196495), caspase-3 (ab13847), cleaved caspase-3 (ab2302), caspase-9 
(ab52298), cleaved caspase-9 (ab2324) were purchased from Abcam (UK).

\section{Animal model establishment and grouping}

Animal model was establishment as previously reported (8). The HIF-1 $\alpha$ lentiviral vector was constructed. The rats were divided into four groups according to the random number table method: the control group, RI/R group, RI/ $\mathrm{R}+$ lentivirus $(\mathrm{LV})$ group, and $\mathrm{RI} / \mathrm{R}+\mathrm{LV}-\mathrm{HIF}-1 \alpha$ group. We used HIF-1 $\alpha$ overexpression or vector control lentivirus $(\mathrm{LV})$ to affect rats recorded as the $\mathrm{RI} / \mathrm{R}+\mathrm{LV}-\mathrm{HIF}-1 \alpha$ group and $\mathrm{RI} / \mathrm{R}+\mathrm{LV}$ group. All rats were anesthetized first and were dissected by midline laparotomy to expose bilateral kidneys. Both sides of the renal pedicle were clamped for 45 minutes and then restored blood flow and reperfusion. After loosening the arterial clamp, if the color of the kidney changed from dark red to bright red gradually, the modeling was determined as successful. After six hours of reperfusion, the indexes were evaluated.

\section{Reverse transcription-polymerase chain reaction (RT-PCR)}

Total RNA of rat myocardial tissues and H9C2 cells was extracted by Trizol agent, and then the absorbance (A260/ A280) of RNA samples was measured with a Nanodrop spectrophotometer. According to the kit instructions, cDNA synthesis and PCR amplification were performed following the following steps: denatured for 5 minutes; (denatured at $94{ }^{\circ} \mathrm{C}$ for 30 seconds, annealed at $55^{\circ} \mathrm{C}$ for 30 seconds, extended at $72{ }^{\circ} \mathrm{C}$ for 30 seconds) amplified for 35 cycles; extended at $72{ }^{\circ} \mathrm{C}$ for 10 minutes; stored at $4{ }^{\circ} \mathrm{C}$. The products were separated by $2 \%$ agarose gel electrophoresis.

\section{Renal function index}

The urine and blood of rats in each group were collected. The proteinuria content in the urine and the levels of creatinine and urea nitrogen in separated serum were detected using an automatic biochemical analyzer.

\section{Hematoxylin and eosin (HE) staining}

The kidney tissues of rats in each group were first fixed with $10 \%$ formaldehyde for 48 hours and embedded in paraffin to make sections into $5 \mathrm{~mm}$ thick. Then the sections were stained with hematoxylin and eosin. The damage of kidney tissue was observed under a fluorescence microscope at a magnification of 400 folds.

\section{TUNEL staining}

Kidney tissue sections of the rat were washed with xylene twice for dewaxing (5 minutes/time), dehydrated using gradient ethanol, blocked with proteinase $\mathrm{K}$ working solution at $37^{\circ} \mathrm{C}$ for 20 minutes, and washed twice with PBS. Followed by adding $50 \mu \mathrm{L}$ of TUNEL reaction solution to each section and react in the dark at $37{ }^{\circ} \mathrm{C}$ for 60 minutes, the sections were counterstained with 4',6-diamidino-2-phenylindole (DAPI) for 10 minutes. The sections could be observed under a fluorescence microscope.

\section{Enzyme-linked immunosorbent assay (ELISA)}

The antigen was dissolved in a $50 \mathrm{mM}$ carbonate coating buffer at $4{ }^{\circ} \mathrm{C}$, which was added into a 96-well microtiter plate $(100 \mu \mathrm{L} /$ well) overnight to coat the antigen. On the next day, discarding the coating solution, the coated plate was washed with $1 \times$ phosphate-buffered saline with Tween 20 (PBST) three times (5 minutes/time), blocked with $1 \%$ bovine serum albumin (BSA) $(150 \mu \mathrm{L} /$ well) for 1 hour. Then they were washed with PBST three times again and added with $100 \mu \mathrm{L}$ of serum at different dilutions and control samples to incubate at $37^{\circ} \mathrm{C}$ for 2 hours. Next, they were washed with PBST five times, added with $100 \mu \mathrm{L}$ of diluted Horse Radish Peroxidase (HRP)-labeled secondary antibody to incubate at $37^{\circ} \mathrm{C}$ for 1 hour, washed with PBST for five times. The plate was added with the coloring agent to develop color for 20 minutes, and the absorbance was measured with a microplate reader.

\section{Western blotting}

The kidney tissues in each group were collected and lysed with lysis buffer holding protease inhibitor for total protein extraction on ice for 25 minutes. After centrifuged at $12,000 \mathrm{r} / \mathrm{minutes}$ for 10 minutes, the supernatant was collected, and the protein content was measured with the Bicinchoninic acid (BCA) kit. After denatured at $100{ }^{\circ} \mathrm{C}$ for 5 minutes, an equal amount of protein samples $(20 \mathrm{mg})$ were loaded into each well and separated by the Sodium dodecylsulphate polyacrylamide gel electrophoresis (SDS-PAGE) gel electrophoresis, then transferred to polyvinylidene fluoride (PVDF) membrane. After blocking, 
A

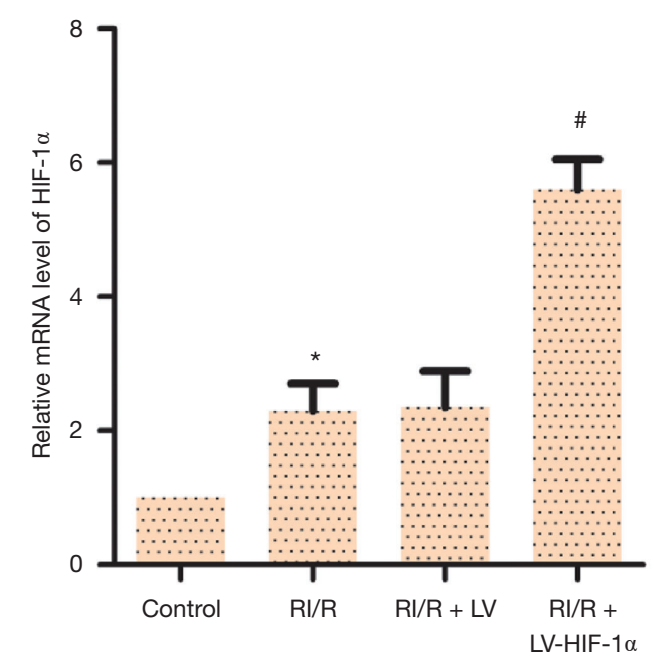

B
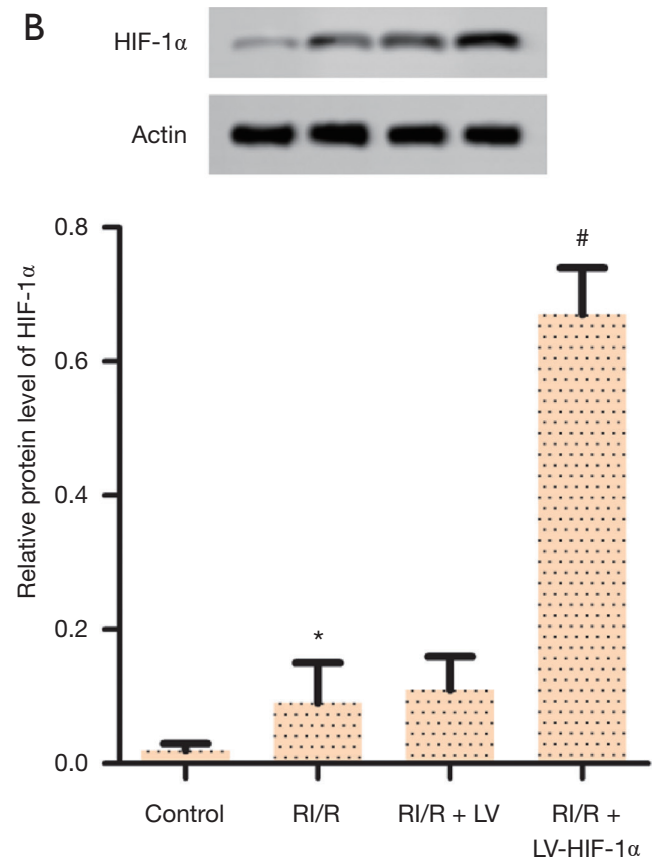

Figure 1 Validate of LV-HIF-1 $\alpha$ transfection. Effect of LV-HIF-1 $\alpha$ on HIF-1 $\alpha$ mRNA (A) and protein (B) expression levels in RI/R rats were tested with RT-PCR and western blotting. * $\mathrm{P}<0.05$ versus control; ${ }^{*}, \mathrm{P}<0.05$ versus RI/R. HIF-1 $\alpha$, hypoxia-inducible factor- $1 \alpha$; RI/R, renal ischemia/reperfusion; $\mathrm{LV}$, lentivirus.

the membrane was incubated with corresponding primary antibody at $4{ }^{\circ} \mathrm{C}$ overnight, washed with TBST buffer, then added with the secondary antibody labeled with horseradish peroxidase at $4{ }^{\circ} \mathrm{C}$ for 2 hours. Enhanced chemiluminescence (ECL) reagent was used for signal detection. Image J software was used for the analysis of the gray value of each band.

\section{Statistical analysis}

SPSS 19.0 software was used for statistical analysis. Normal distribution measurement data was expressed as $\bar{x} \pm s$ and analyzed using a $t$-test. $\mathrm{P}<0.05$ was considered statistically significant.

\section{Results}

\section{HIF-1a overexpression increases $m R N A$ and protein levels of HIF-1a in RI/R rats}

To validate the effects of HIF-1 $\alpha$ in RI/R, LV-HIF- $1 \alpha$ was established and transfected into SD rats once daily for one week before R/IR was established. The renal tissues were collected for testing. The results of RT-qPCR and Western blotting detection showed that compared with the RI/R + LV group, the expression of HIF- $1 \alpha$ in the RI/R + LV-HIF$1 \alpha$ group increased significantly both at the mRNA and protein levels (Figure $1, \mathrm{P}<0.05$ ). The results supported that HIF- $1 \alpha$ overexpression with LV-HIF- $1 \alpha$ increases mRNA and protein levels of HIF- $1 \alpha$ in RI/R rats.

\section{HIF-1a overexpression reduces proteinuria, urea nitrogen and serum creatinine levels in RI/R rats}

The overall effects of LV-HIF-1 $\alpha$ on renal injury was then evaluated. By detecting the proteinuria, urea nitrogen, and serum creatinine levels of the rats in each group with the kits, the results indicated that compared with the control group, the proteinuria (Figure $2 A$ ), urea nitrogen (Figure 2B), and serum creatinine (Figure 2C) levels of the RI/R group were significantly increased $(\mathrm{P}<0.05)$. Compared with the RI/R + LV group, the proteinuria, urea nitrogen, and serum creatinine levels of the RI/R + LV-HIF$1 \alpha$ group were significantly decreased $(\mathrm{P}<0.05$, Figure 2$)$. The results indicated the protective role of LV-HIF-1 $\alpha$ in $\mathrm{RI} / \mathrm{R}$ rats. 
A

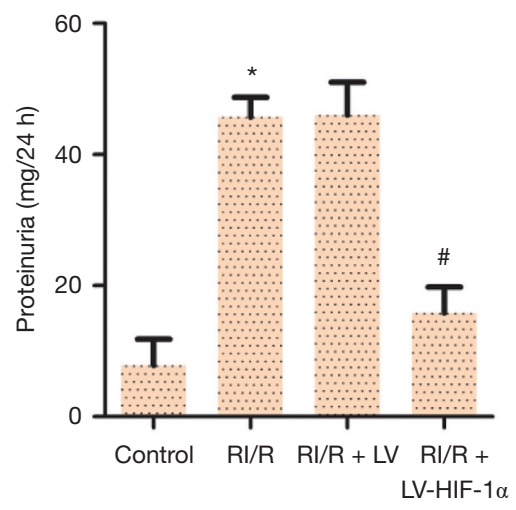

B

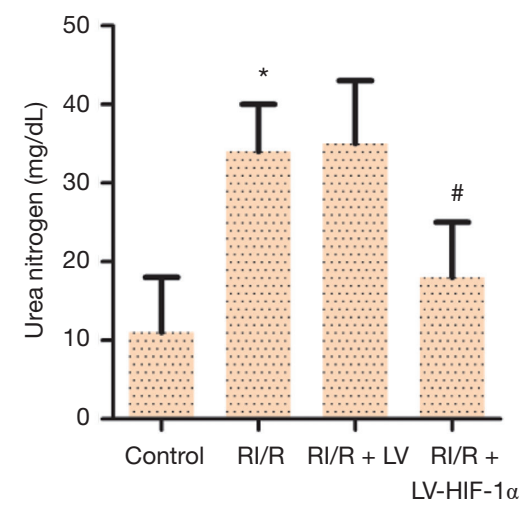

C

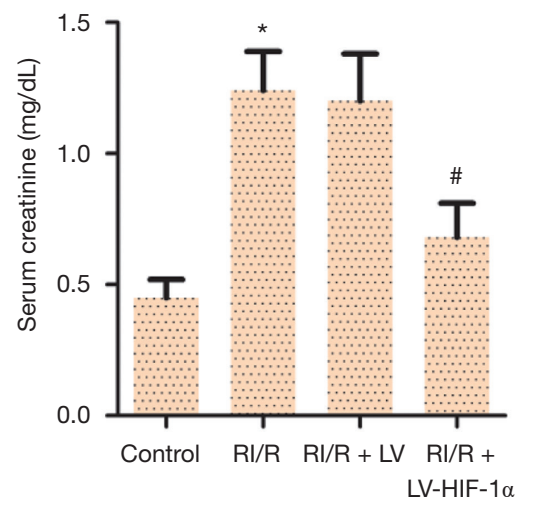

Figure 2 Effect of LV-HIF-1 $\alpha$ on renal function in RI/R rats. Proteinuria content in the urine (A) and the levels of creatinine (B) and urea nitrogen $(\mathrm{C})$ in separated serum were detected using an automatic biochemical analyzer in $\mathrm{RI} / \mathrm{R}$ rats. * $\mathrm{P}<0.05$ versus control; *, $\mathrm{P}<0.05$ versus RI/R. HIF-1 $\alpha$, hypoxia-inducible factor-1 $\alpha$; RI/R, renal ischemia/reperfusion; LV, lentivirus.

\section{HIF-1a overexpression improves the degree of pathological damage in $R I / R$ rats}

To further validate the protective role of LV-HIF-1 $\alpha$ in RI/R rats, HE and TUNEL staining was employed in renal sections. HE staining was used for detecting the pathological damage in each group. The result showed that the kidney tissue structure and glomerular cell morphology of the control group showed no abnormalities. In contrast, the renal tissue pathology of the RI/R group and the RI/ $\mathrm{R}+\mathrm{LV}$ group changed obviously, including the reduced number of glomeruli, edema, expansion, and vacuolation of the kidney tubule, inflammatory cell infiltration, fibrosis, cell congestion, visible protein casts in some areas, which showed apparent renal tissue pathological damage. However, the degree of pathological damage in the RI/R $+\mathrm{LV}$-HIF- $1 \alpha$ group was significantly improved compared with the RI/R + LV group (Figure 3A). TUNEL staining was performed to detect the apoptosis of rats in each group, and the nucleus of TUNEL-positive cells was stained darkly brown. Compared with the control group, the apoptosis rate of the RI/R group was significantly increased $(\mathrm{P}<0.05)$. While compared with the RI/R + LV group, the apoptosis rate in the RI/R + LV-HIF- $1 \alpha$ group was significantly decreased $(\mathrm{P}<0.05$, Figure $3 B)$. The results supported the renal protective role of $\mathrm{LV}-\mathrm{HIF}-1 \alpha$ on RI/R rats.

\section{HIF-1a overexpression decreases the levels of $I L-6, I L-1 \beta$, and TNF- $\alpha$, and increases the level of $I L-10$}

To explore the underlined mechanism of action for LV-HIF-
$1 \alpha$ in alleviating RI/R, serum levels of typical inflammatory cytokines were tested. The levels of IL-6 (Figure $4 A$ ), IL-1 $\beta$ (Figure 4B), TNF- $\alpha$ (Figure 4C), and IL-10 (Figure $4 D$ ) in each group of rats was detected by ELISA kit. The results showed that compared with the control group, the IL6 , IL-1 $\beta$, and TNF- $\alpha$ levels in the RI/R group increased significantly $(\mathrm{P}<0.05)$. Compared with the $\mathrm{RI} / \mathrm{R}+\mathrm{LV}$ group, the IL- 6 , IL- $1 \beta$, and TNF- $\alpha$ levels in the RI/R + LVHIF-1 $\alpha$ group were significantly decreased $(\mathrm{P}<0.05)$, and IL-10 level was significantly increased $(\mathrm{P}<0.05)$. These supported that $\mathrm{LV}$-HIF-1 $\alpha$ inactivated the inflammation in $\mathrm{RI} / \mathrm{R}$ rats.

\section{HIF-1 1 overexpression reduces the ratio of cleaved caspase-3/caspase-3, cleaved caspase-9/caspase-9, Bax/ Bcl-2 in RI/R rats}

Apoptosis was further investigated to show the mechanism of action for LV-HIF- $1 \alpha$ in RI/R rats. The protein expression levels of caspase-3, caspase-9, Bax, and Bcl-2 in each group were detected by Western blotting. The results showed that compared with the control group, the ratio of cleaved caspase-3/caspase-3, cleaved caspase- $9 /$ caspase- 9 , and $\mathrm{Bax} / \mathrm{Bcl}-2$ in the RI/R group increased significantly $(\mathrm{P}<0.05)$. However, compared with the $\mathrm{RI} / \mathrm{R}+\mathrm{LV}$ group, the ratio of cleaved caspase- $3 /$ caspase- 3 , cleaved caspase- $9 /$ caspase-9, Bax/Bcl-2 in the RI/R + LV-HIF- $1 \alpha$ group was significantly decreased $(\mathrm{P}<0.05$, Figure $5 A, B)$. The results showed that LV-HIF-1 $\alpha$ inhibited the apoptosis of renal cells in $\mathrm{RI} / \mathrm{R}$ rats. 
A

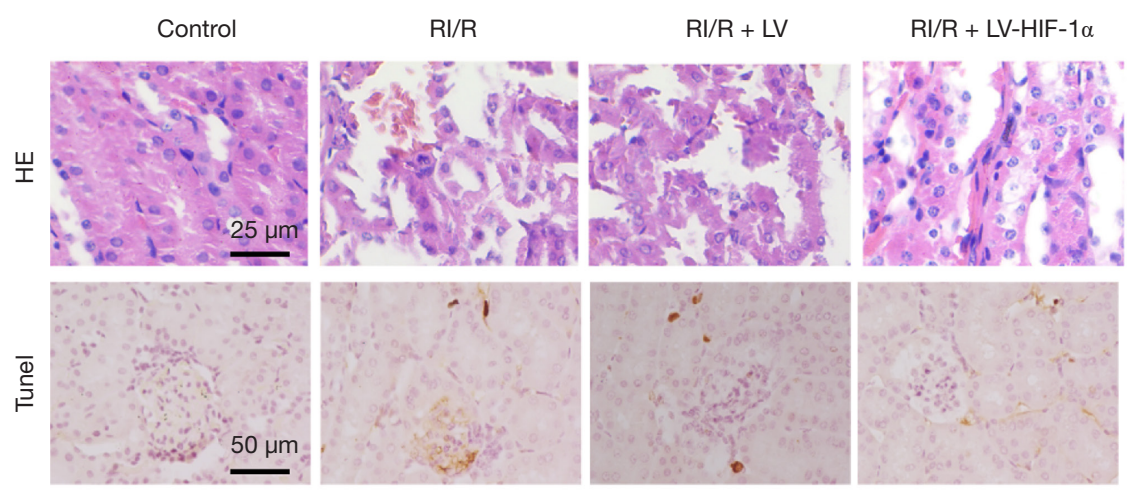

B

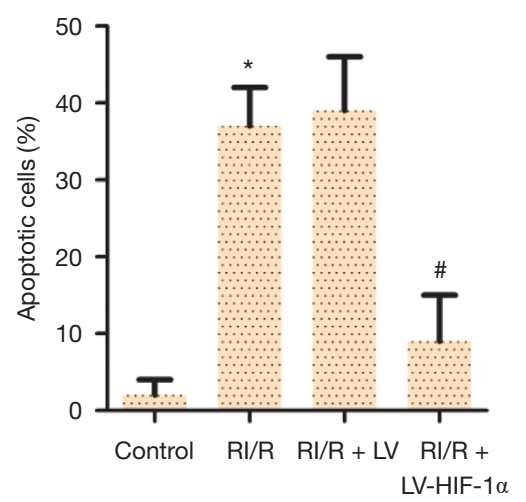

Figure 3 Effect of LV-HIF-1 $\alpha$ on pathological injury degree in RI/R rats. (A) Renal injury indicated with HE and TUNEL staining. (B) Apoptotic renal cells were counted. *, $\mathrm{P}<0.05$ versus control; * $\mathrm{P}<0.05$ versus RI/R. HIF-1 $\alpha$, hypoxia-inducible factor-1 $\alpha$; RI/R, renal ischemia/reperfusion; LV, lentivirus.

\section{Discussion}

As the most common cause of acute kidney injury, RI/R injury can result in a sharp decline in renal function (9). Because RI/R injury has a complex development and changing process, its pathophysiology mechanisms have not yet been sufficiently elaborated, and currently, there are no effective prevention and intervention ways for RI/R injury (10). Related studies have shown that HIF-1 $\alpha$ could be activated during reperfusion after renal ischemia, which is crucial for the survival of proximal tubule cells (11). HIF- $1 \alpha$ may help repair kidney tissues via regulating inflammation reactions after ischemia/reperfusion (12). Results in this study showed that the overexpression of HIF- $1 \alpha$ could improve the pathological tissue damage of $\mathrm{RI} / \mathrm{R}$ rats.

$\mathrm{RI} / \mathrm{R}$ can cause severe nephron damage, such as decrease of glomerular filtration rate and damage of renal tubular epithelial cells, resulting in weakened renal tubular reabsorption, which, clinically, often manifests the increase of urea nitrogen and creatinine levels in serum, and the appearance of proteinuria symptoms (13). This study found that HIF-1 $\alpha$ overexpression had the effects of reducing proteinuria, urea nitrogen, and serum creatinine levels in RI/R rats, suggesting that HIF- $1 \alpha$ overexpression has excellent protective effects on $\mathrm{RI} / \mathrm{R}$ rats via alleviating the damage of glomeruli and renal tubules caused by ischemia/ reperfusion.

Inflammation is a prominent feature of ischemia/ reperfusion injury, which is characterized by leukocyte infiltration and renal tubular injury (14). Studies have shown that during RI/R process, kidney tissues will produce a variety of inflammatory mediators formed after ischemia, such as TNF- $\alpha$ and IL- $1 \beta$, which are strictly related to ischemic injury (15). During RI/R injury, the inflammatory reactions are activated, and many proinflammatory cytokines are released. The expression of adhesion molecules on the surface of vascular endothelial cells and leukocytes are increased, resulting in the activation of leukocytes to damage the tissues by adhesion to vascular endothelial cells (16). In the inflammatory response network, IL-6, IL- $1 \beta$, and TNF- $\alpha$ are important proinflammatory cytokines. TNF- $\alpha$ increases the adhesion of vascular endothelial cells to neutrophils by up-regulating the expression of vascular endothelial adhesion factors, which leads to effusion and migration of inflammatory cells and causing the block of microcirculation of body tissues and damage. IL- 6 and IL- $1 \beta$ can promote granulocytemediated inflammation and neutrophil activation, which may aggravate the degree of kidney damage, while IL-10 plays a protective role against RI/R injury (17). This study found that HIF- $1 \alpha$ overexpression showed the effects of decreasing the levels of IL- 6 , IL- $1 \beta$, TNF- $\alpha$ and increasing the level of IL-10 in RI/R rats, which suggests that HIF$1 \alpha$ overexpression improves RI/R injury by regulating the release of inflammatory factors.

Apoptosis is one of the programmed ways of cell death with three main pathways, including exogenous apoptosis pathways, endogenous apoptosis pathways, and endoplasmic reticulum stress pathways (18). The endogenous apoptosis pathways are also called a mitochondrial pathway, which is 
A

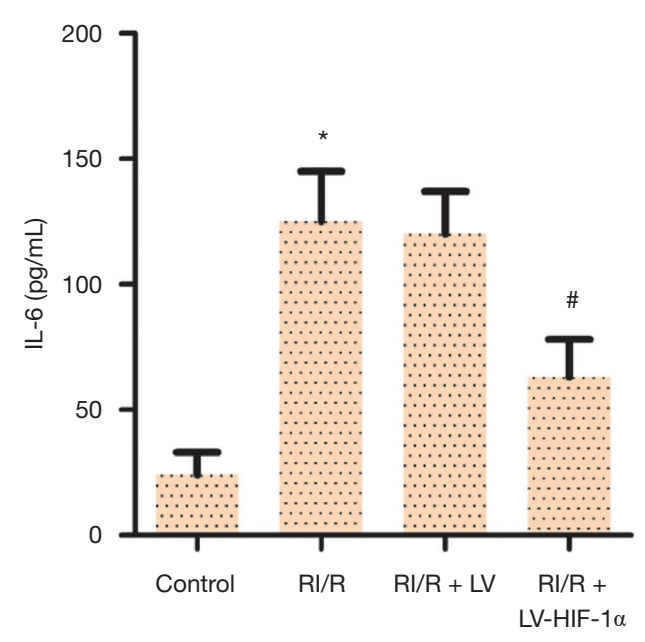

C

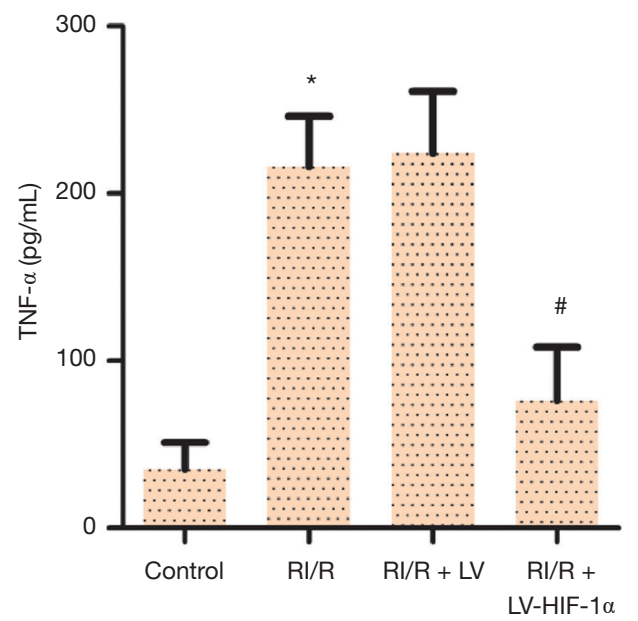

B

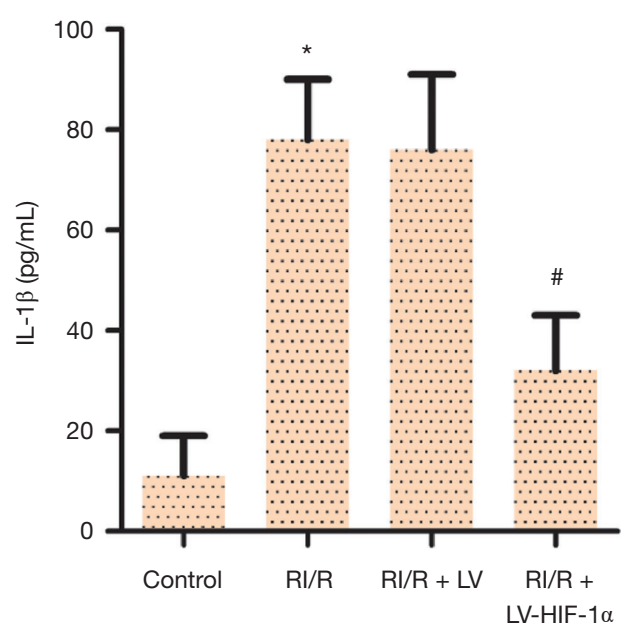

$\mathrm{D}$

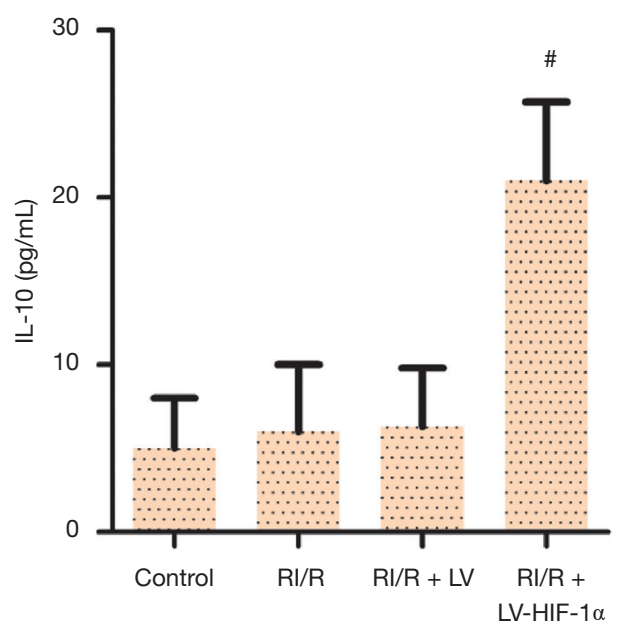

Figure 4 Effect of LV-HIF-1 $\alpha$ on typical inflammatory cytokines in RI/R rats. Serum levels of IL-6 (A), IL-1 $\beta$ (B), TNF- $\alpha$ (C) and IL-10 (D) were investigated with ELISA. *, $\mathrm{P}<0.05$ versus control; \#, $\mathrm{P}<0.05$ versus RI/R. HIF-1 $\alpha$, hypoxia-inducible factor- $1 \alpha$; RI/R, renal ischemia/reperfusion; LV, lentivirus.

crucial to support cell physiology or pathology. Apoptosis is one way of tissue and organ damage during ischemia/ reperfusion, which happens in the renal tubular epithelial cells with the most apparent apoptosis in the distal tubular epithelial cells (19). The caspase family proteins are critical elements in apoptosis, which can regulate apoptosis by interacting with many proteins and cytokines. Among the caspase family members, excessive activation of caspase-9 and caspase- 3 in the apoptosis cascade pathway will promote apoptosis (20). The anti-apoptotic member Bcl-2 can prevent apoptosis, while the pro-apoptotic member Bax, in the outer mitochondrial membrane or cytoplasm, is oligomerized under stress to promote cytokines release from mitochondrial, triggering apoptosis (21). In this study, it was found that HIF- $1 \alpha$ overexpression reduced the ratios of cleaved caspase-3/caspase-3, cleaved caspase-9/ caspase-9, and $\mathrm{Bax} / \mathrm{Bcl}-2$ in $\mathrm{RI} / \mathrm{R}$ rats, suggesting that the overexpression of $\mathrm{HIF}-1 \alpha$ can protect RI/R injury by regulating mitochondrial apoptosis pathway.

In summary, the overexpression of HIF-1 $\alpha$ has protective 
A

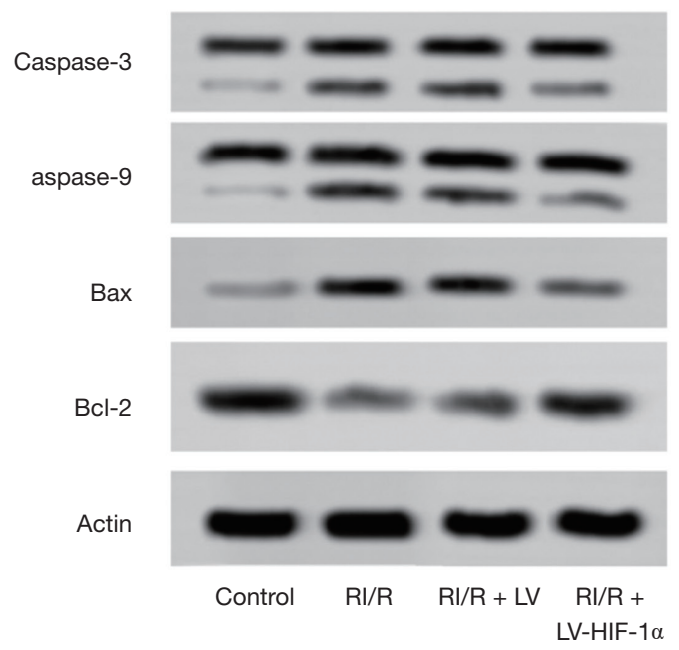

B

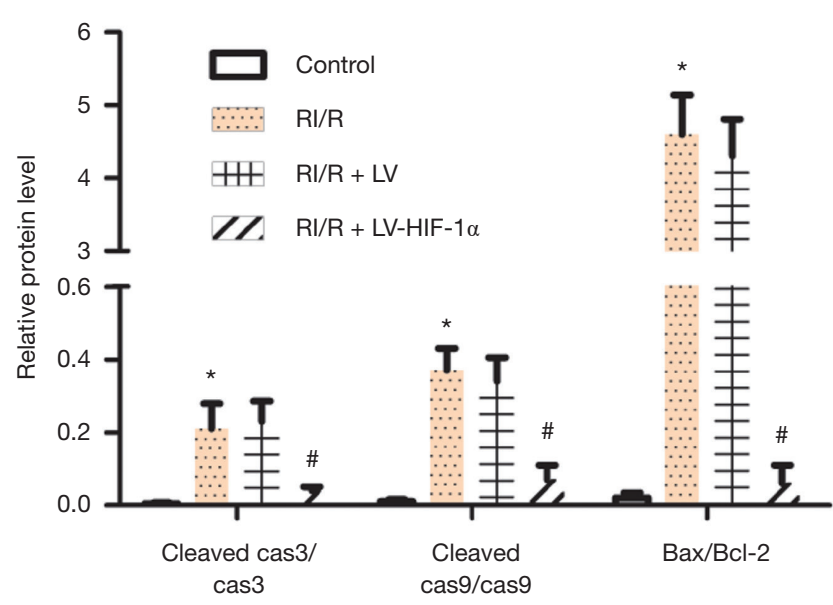

Figure 5 Effect of LV-HIF-1 $\alpha$ on apoptotic proteins in RI/R rats. (A) Caspase-3, Caspase-9, Bax and Bcl-2 proteins were tested with western blotting. (B) Relative protein levels of Caspase-3, Caspase-9, Bax and Bcl-2 were semi-quantified with Image J. *, $\mathrm{P}<0.05$ versus control; ", $\mathrm{P}<0.05$ versus RI/R. HIF-1 $\alpha$, hypoxia-inducible factor- $1 \alpha$; RI/R, renal ischemia/reperfusion; $\mathrm{LV}$, lentivirus.

effects on RI/R rats by improving renal function, inhibiting the release of inflammatory factors, and reducing the expression of apoptosis proteins during RI/R.

\section{Acknowledgments}

Funding: None.

\section{Footnote}

Reporting Checklist: The authors have completed the ARRIVE reporting checklist. Available at http://dx.doi. org/10.21037/tau-20-918

Data Sharing Statement: Available at http://dx.doi. org/10.21037/tau-20-918

Conflicts of Interest: All authors have completed the ICMJE uniform disclosure form (available at http://dx.doi. org/10.21037/tau-20-918). The authors have no conflicts of interest to declare.

Ethical Statement: The authors are accountable for all aspects of the work in ensuring that questions related to the accuracy or integrity of any part of the work are appropriately investigated and resolved. The animal experiments in this study were approved by the Ethics
Committee of the Xiangyang No. 1 People's Hospital (SYXK (Hubei) 2016-0031). Each experiment was performed in accordance with the Guide for the Care and Use of Laboratory Animals, 8th edition, published by the National Research Council (US) Committee.

Open Access Statement: This is an Open Access article distributed in accordance with the Creative Commons Attribution-NonCommercial-NoDerivs 4.0 International License (CC BY-NC-ND 4.0), which permits the noncommercial replication and distribution of the article with the strict proviso that no changes or edits are made and the original work is properly cited (including links to both the formal publication through the relevant DOI and the license). See: https://creativecommons.org/licenses/by-nc-nd/4.0/.

\section{References}

1. Xie GL, Zhu L, Zhang YM, et al. Change in iron metabolism in rats after renal ischemia/reperfusion injury. PloS One 2017;12:e0175945.

2. Nicholson ML, Pattenden CJ, Barlow AD, et al. A double blind randomized clinical trial of remote ischemic conditioning in live donor renal transplantation. Medicine (Baltimore) 2015;94:e1316.

3. Vo AA, Zeevi A, Choi J, et al. A phase I/II placebocontrolled trial of C1-inhibitor for prevention of 
antibody-mediated rejection in HLA sensitized patients. Transplantation 2015;99:299-308.

4. Sethi, K, Rao, K, Bolton, D, et al. Targeting HIF-1 $\alpha$ to Prevent Renal Ischemia-Reperfusion Injury: Does It Work? Int J Cell Biol 2018:9852791.

5. Ye XB, Xiong LS, Wu J, et al. The role of hypoxiainducible factor- $1 \alpha(\mathrm{HIF}-1 \alpha)$ in the pathogenesis of prostate cancer. China Modern Doctor 2018;56:25-7.

6. Semenza GL. Oxygen sensing, hypoxia-inducible factors, and disease pathophysiology. Annu Rev Pathol 2014;9:47-71.

7. Knudsen AR, Kannerup AS, Gronbaek H, et al. Effects of ischemic pre-and postconditioning on hif-1alpha, vegf and tgf-beta expression after warm ischemia and reperfusion in the rat liver. Comp Hepatol 2011;10:3.

8. Yang J, Hu YL, Zheng MD, et al. Protective effect of allitridi on oxidative stress in the rats with renal ischemicReperfusion Injury. Journal of Medical Research 2017;46:108-11.

9. Yu SQ, Ma S, Wang DH. Activation of TRPV1 prevents salt-induced kidney damage and hypertension after renal ischemia-reperfusion injury in rats. Kidney Blood Press Res 2018;43:1285-96.

10. Sun $W$, Zhu Q, Yan L, et al. Mesenchymal stem cells alleviate acute kidney injury via miR-107-mediated regulation of ribosomal protein S19. Ann Transl Med 2019;7:765.

11. Conde E, Alegre L, Blanco-Sánchez I, et al. Hypoxia inducible factor 1-alpha (HIF-1 alpha) is induced during reperfusion after renal ischemia and is critical for proximal tubule cell survival. PLoS One 2012; 7:e33258.

12. Hill P, Shukla D, Tran MG, et al. Inhibition of hypoxia inducible factor hydroxylases protects against renal ischemia-reperfusion injury. J Am Soc Nephrol 2008;19:39-46.

13. Barbar SD, Dargent A, Quenot JP. Initiation of renal

Cite this article as: Li X, Chen W, Feng J, Zhao B. The effects of HIF- $1 \alpha$ overexpression on renal injury, immune disorders and mitochondrial apoptotic pathways in renal ischemia/ reperfusion rats. Transl Androl Urol 2020;9(5):2157-2165. doi: 10.21037/tau-20-918 replacement therapy in patients with septic acute kidney injury: right timing or right patient? Ann Transl Med 2019;7:598.

14. Xie LB, Chen X, Chen B, et al. Protective effect of bone marrow mesenchymal stem cells modified with klotho on renal ischemia-reperfusion injury. Ren Fail 2019;41:175-82.

15. Du X, Hu X, Wei J. Anti-inflammatory effect of exendin-4 postconditioning during myocardial ischemia and reperfusion. Mol Biol Rep 2014;41:3853-7.

16. Sun YW, Peng KN, Li YY, et al. Effects of Panax notoginseng saponins pretreatment on expressions of NF-kappa Bp65 and IL-1 $\beta$ in rats with renal ischemia reperfusion injury. Pharmacology and Clinics of Chinese Materia Medica 2017;33:58-62.

17. Kolachala VL, Palle S, Shen M, et al. Loss of L-selectin guided $\mathrm{CD} 8+$, but not CD4+, cells protects against ischemia reperfusion injury in a steatotic liver. Hepatology 2017;66:1258-74.

18. Modi S, Kir D, Banerjee S, et al. Control of apoptosis in treatment and biology of pancreatic cancer. J Cell Biochem 2016;117:279-88.

19. Zhong D, Wang H, Liu M, et al. Ganoderma lucidum polysaccharide peptide prevents renal ischemia reperfusion injury via counteracting oxidative stress. Sci Rep 2015;5:16910.

20. Chantal BH, Anne J, Laurence L, et al. Acute cytotoxicity of mira-1/nsc19630, a mutant p53-reactivating small molecule, against human normal and cancer cells via a Caspase-9-dependent apoptosis. Cancer Lett 2015;359:211-7.

21. Czabotar PE, Guillaume L, Andreas S, et al. Control of apoptosis by the bcl-2 protein family: Implications for physiology and therapy. Nat Rev Mol Cell Biol 2014;15:49-63. 